\title{
Tau Imaging: New Era of Neuroimaging for Alzheimer's Disease
}

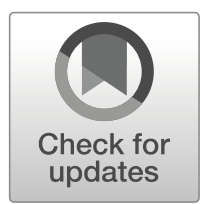

\author{
Jae Seung Kim ${ }^{1}$
}

Received: 9 July 2020 / Revised: 18 July 2020 / Accepted: 22 July 2020 / Published online: 27 July 2020

(C) Korean Society of Nuclear Medicine 2020

The US FDA recently approved Tauvid (F-18 flortaucipir), the first radioligand indicated for positron emission tomography (PET) imaging of the brain to estimate the density and distribution of aggregated tau neurofibrillary tangles (NFTs), one of the two neuropathological hallmarks of Alzheimer's disease (AD). This approval is based on evidence for 1921 patients in 19 clinical trials conducted at 322 sites in the USA, Australia, Belgium, Canada, France, Japan, Netherlands, and Poland [1].

$\mathrm{AD}$ is the most common etiology of dementia. In 2017, the prevalence of dementia among Koreans over 65 years was estimated to be $10.2 \%$, and the number of dementia patients is estimated to be 724,857 . The number of dementia patients will double every 17 years and reach almost 3 million in 2050 [2]. AD is characterized by the deposition of beta amyloid $(\mathrm{A} \beta)$ plaques and neurofibrillary tangles, which are misfolded aggregates of hyperphosphorylated tau proteins in the brain [3]. AD can only be definitively diagnosed by postmortem pathologic evaluation of the patient's brain. For A $\beta$ pathology, there are three PET imaging radioligands (florbetaben, flutemetamol, and florapronol) that are approved and clinically used in South Korea [4]. Several clinical stages have been defined in AD, including preclinical, prodromal, and dementia. As $A \beta$ radioligands were developed, specific diagnostic criteria integrating amyloid PET imaging was recently proposed to better define these stages [5]. It seems, however, that amyloid PET imaging alone does not distinguish the symptomatic stages of AD. Tau protein, which plays another major role in the pathogenesis of $\mathrm{AD}$, is a microtubule-associated protein (MAP) which is essential to neuronal stability and functioning [6]. Abnormal hyperphosphorylation of tau causes aggregated tau NFTs in the AD brain. Abnormal tau aggregation occurs also in other tauopathies (corticobasal degeneration,

Jae Seung Kim

jaeskim@amc.seoul.kr

1 Department of Nuclear Medicine, Asan Medical Center, University of Ulsan College of Medicine, 88, Olympic-ro 43-gil, Songpa-Gu, Seoul 05505, Republic of Korea frontotemporal dementia with parkinsonism linked to chromosome 17, Pick's disease, progressive supranuclear palsy, and chronic traumatic encephalopathy) [7].

Tauvid (F-18 flortaucipir, known as AV-1451 and T-807) is one of the so-called first-generation radioligands (e.g., F-18 THK5351 and C-11 PBB3) and exhibits high binding affinity for the paired helical filament (PHF)-tau and high binding selectivity for tau over $\mathrm{A} \beta$ in $\mathrm{AD}$ brains [8]. Postmortem pathology studies have shown that flortaucipir binding correlates with postmortem NFT Braak staging in AD brains [9]. Human PET studies showed preferential uptake in the inferior temporal and posterior parietal cortices of AD patients [10]. The amount and area of abnormal uptake significantly correlated with the clinical severity of dementia. Cortical uptake of flortaucipir was also found in some of the preclinical AD patients but rarely in $A \beta$-negative patients [11]. Age-related increase in flortaucipir uptake was noted in medial temporal cortex of $A \beta$-negative, normal subjects, suggesting the existence of age-related tau deposition in old aged population, which is pathologically classified as primary age-related tauopathy [12]. However, it has not been confirmed whether flortaucipir retention in medial temporal cortex reflects agerelated tau pathology in cognitively normal subjects. The cortical distribution of flortaucipir was varied in atypical AD cases and was reported to reflect clinical and neuroanatomical diversity [13]. Flortaucipir retention was correlated with regional gray matter atrophy in MCI and AD patients [14]. Flortaucipir binding in the parahippocampal gyrus and neocortex was shown to correlate with CSF-tau levels and strongly correlated with neurodegeneration and cognitive decline [15].

Although the first-generation radioligands such as flortaucipir for tau imaging were promising, some off-target binding led to the optimization of the binding properties and development of the second-generation radioligands followed (i.e., F-18 PI-2620, F-18 MK-6240, F-18 GTP1, F-18 PMPBB3, F-18 JNJ-311). In flortaucipir PET studies, off-target binding was noted in the striatum, midbrain, choroid plexus, and meninges. In analysis of autopsy, flortaucipir has been shown to bind iron-related regions, the substantia nigra, 
calcifications in the choroid plexus, and leptomeningeal melanin [16]. Increased uptake of flortaucipir in the substantia nigra and the meninges is considered to be associated with neuromelanin and melanocyte binding. Another off-target binding site is the choroid plexus located in the temporal lobe. Therefore, high radioactivity in the choroid plexus can spill over into adjacent hippocampus and cause a misinterpretation of PET image.

Recent advances in the development of tau PET radioligands have enabled the noninvasive monitoring of PHF-tau accumulation in aging and AD brains. However, information on the speed and direction of tau pathology progression during the course of aging and $\mathrm{AD}$ is still limited. Current ongoing longitudinal studies may help clarify the natural history of PHF-tau formation. Tau PET may also be useful in the therapeutic trials targeting tau pathology, both for subject screening and as a surrogate imaging biomarker. Therefore, it is important to establish methods for accurate quantification of PET images and visual interpretation as in amyloid imaging [17]. In addition, several studies have shown a poor association of flortaucipir binding with non-AD tauopathies that have preferential accumulation of either $3 \mathrm{R}$ or $4 \mathrm{R}$ tau [18]. Therefore, the development of new radioligands capable of selectively imaging $3 \mathrm{R}$ or $4 \mathrm{R}$ tauopathies will be needed.

\section{Compliance with Ethical Standards}

Conflict of Interest Jae Seung Kim declares no conflict of interest.

Ethical Approval This work does not contain any studies with human participants or animals performed by the author.

Informed Consent Not applicable.

\section{References}

1. FDA news release. FDA approves first drug to image tau pathology in patients being evaluated for Alzheimer's disease. FDA. 2020. https://www.fda.gov/news-events/press-announcements/fdaapproves-first-drug-image-tau-pathology-patients-being-evaluatedalzheimers-disease. Accessed: 15 July 2020.

2. Ministry of Health \& Welfare. 2012 National study on the prevalence of dementia in Korean elders. 8th ed. Ministry of Health \& Welfare; Korea: 2012.

3. Braak H, Braak E. Neuropathological stageing of Alzheimerrelated changes. Acta Neuropathol. 1991;82:239-59.
4. Jeong J, Jeong YJ, Park KW, Kang D-Y. Correlation of early-phase F-18 florapronol PET with F-18 FDG PET in Alzheimer's disease and normal brain. Nucl Med Mol Imaging. 2019;53:328-33.

5. Dubois B, Hampel H, Feldman HH, Scheltens P, Aisen P, Andrieu $\mathrm{S}$, et al. Preclinical Alzheimer's disease: definition, natural history, and diagnostic criteria. Alzheimers Dement. 2016;12(3):292-323.

6. Goedert M, Crowther RA, Garner CC. Molecular characterization of microtubule-associated proteins tau and MAP2. Trends Neurosci. 1991;14:193-9.

7. Villemagne VL, Fodero-Tavoletti MT, Masters CL, Rowe CC. Tau imaging: early progress and future directions. Lancet Neurol. 2015;14:114-24.

8. Xia CF, Arteaga J, Chen G, Gangadharmath U, Gomez LF, Kasi D, et al. $[18 \mathrm{~F}] \mathrm{T} 807$, a novel tau positron emission tomography imaging agent for Alzheimer's disease. Alzheimers Dement. 2013;9: 666-76.

9. Marquie M, Siao Tick Chong M, Anton-Fernandez A, Verwer EE, Saez-Calveras N, Meltzer AC, et al. [F-18]-AV-1451 binding correlates with postmortem neurofibrillary tangle Braak staging. Acta Neuropathol. 2017;134:619-28.

10. Johnson KA, Schultz A, Betensky RA, Becker JA, Sepulcre J, Rentz D, et al. Tau positron emission tomographic imaging in aging and early Alzheimer disease. Ann Neurol. 2016;79:110-9.

11. Pontecorvo MJ, Devous MD Sr, Navitsky M, Lu M, Salloway S, Schaerf FW, et al. Relationships between flortaucipir PET tau binding and amyloid burden, clinical diagnosis, age and cognition. Brain. 2017;140:748-63.

12. Crary JF, Trojanowski JQ, Schneider JA, Abisambra JF, Abner EL, Alafuzoff I, et al. Primary age-related tauopathy (PART): a common pathology associated with human aging. Acta Neuropathol. 2014;128:755-66.

13. Ossenkoppele R, Schonhaut DR, Scholl M, Lockhart SN, Ayakta N, Baker SL, et al. Tau PET patterns mirror clinical and neuroanatomical variability in Alzheimer's disease. Brain. 2016;139:155167.

14. Nasrallah IM, Chen YJ, Hsieh MK, Phillips JS, Ternes K, Stockbower GE, et al. 18F-Flortaucipir PET/MRI correlations in nonamnestic and amnestic variants of Alzheimer disease. J Nucl Med. 2018;59:299-306.

15. Gordon BA, Friedrichsen K, Brier M, Blazey T, Su Y, Christensen $\mathrm{J}$, et al. The relationship between cerebrospinal fluid markers of Alzheimer pathology and positron emission tomography tau imaging. Brain. 2016;139:2249-60.

16. Lowe VJ, Curran G, Fang P, Liesinger AM, Josephs KA, Parisi JE, et al. An autoradiographic evaluation of AV-1451 tau PET in dementia. Acta Neuropathol Commun. 2016;4:58.

17. Kim J-Y, Suh HY, Ryoo HG, Oh D, Choi H, Paeng JC, et al. Amyloid PET quantification via end-to-end training of a deep learning. Nucl Med Mol Imaging. 2019;53:340-8.

18. Marquié M, Normandin MD, Meltzer AC, Siao Tick Chong M, Andrea NV, Antón-Fernández A, et al. Pathological correlations of [F-18]-AV-1451 imaging in non-alzheimer tauopathies. Ann Neurol 2017;81:117-128.

Publisher's Note Springer Nature remains neutral with regard to jurisdictional claims in published maps and institutional affiliations. 